\title{
Bundle-Connection Pairs and Loop Group Representations
}

\author{
P. Gibilisco
}

UDC 514.17

\begin{abstract}
Let $M$ be a connected differentiable manifold. Denote by $\Omega_{m}(M)$ the space of $H^{1}$-loops based at a fixed point $m \in M$. Associated to $\Omega_{m}(M)$ one has $\widetilde{\Omega}_{m}(M)$, the group of unparameterized loops. Given a bundle-connection pair $(E, \nabla)$ over $M$ with fiber the finite-dimensional vector space $V$ and structure group $G \subset G L(V)$ we get (up to equivalence) a smooth representation of $\widetilde{\Omega}_{m}(M)$ in $G$ given by the parallel transport operator $P^{\nabla}$. It is possible to find in the literature several versions of the converse theorem, namely: all (smooth) representations of $\widetilde{\Omega}_{m}(M)$ arise in the above described way from a bundle-connection pair. It is shown in the present paper that the correct setting for this theorem is the theory of induced representations for groupoids.
\end{abstract}

KEY WORDS: connection, loop group, differentiable manifold, bundle, induced representation.

\section{§o. Introduction}

Let $M$ be a connected differentiable manifold and let $m$ be an arbitrary point in $M$. Associated to $M$ we have $\mathcal{P}(M)$, the set of $H^{1}$-curves, $\mathcal{P}_{m}(M)$, the set of curves starting at $m, \Omega_{m}(M)$, the set of loops based at $m$, and $\Omega_{m}^{o}(M)$, the set of loops based at $m$ and equivalent to $m$ by homotopy. After choosing a suitable equivalence relation on $\mathcal{P}(M)$ one obtains two groupoids $\widetilde{\mathcal{P}}(M), \widetilde{\mathcal{P}}_{m}(M)$, the loop group $\widetilde{\Omega}_{m}(M)$ and the restricted loop group $\widetilde{\Omega}_{m}^{o}(M)$ canonically associated to the manifold $M$. One may add to this list the fundamental group $\pi_{1}(M)$. In some sense the theory of bundle-connection pairs over $M$ can be expressed in terms of the representation theory of the groups (groupoids) $\pi_{1}(M), \widetilde{\Omega}_{m}^{o}(M), \widetilde{\Omega}_{m}(M)$, $\widetilde{\mathcal{P}}(M)$.

Let us first discuss two results that are "extreme" in some sense: one is purely "local" and the other is purely "global" in character. One of the oldest results emphasizing the group-theoretical character of the theory of connections is the Ambrose-Singer theorem. This theorem states that two "local" objects associated to a connection are isomorphic. Suppose one has a bundle-connection pair $(E, \nabla)$ over $M$ and let $P^{\nabla}$ be the associated parallel transport operator. The operator $P^{\nabla}$ gives a representation of the loop group $\widetilde{\Omega}_{m}(M)$ (and therefore of the restricted loop group $\widetilde{\Omega}_{m}^{o}(M)$ ) in the linear group of the fiber $E_{m}$. The images of these representations are the holonomy group $\operatorname{Hol}_{m}(\nabla)$ and the restricted holonomy group $\mathrm{Hol}_{m}^{\circ}(\nabla)$ respectively. For a fixed point $m$, one may consider $\operatorname{hol}_{m}(\nabla)$, the holonomy Lie algebra"generated" by the curvature of $\nabla$ at $m$ (see [1-2]). The content of the Ambrose-Singer theorem is that $\operatorname{Lie}\left(\mathrm{Hol}_{m}^{\circ}(\nabla)\right)=\operatorname{hol}_{m}(\nabla)$. On the contrary, let us consider a bundle equipped with a (locally) flat connection. The parallel transport operator can now be used to produce a representation of the fundamental group $\pi_{1}(M)$. Vice versa, it is well known that given a representation of $\pi_{1}(M)$, it is possible to reconstruct a bundle equipped with a connection with zero curvature ([3-5]).

Now we recall two results regarding arbitrary connections. Given a bundle-connection pair one has, again using parallel transport, a representation of the groupoid $\widetilde{\mathcal{P}}(M)$ in the groupoid of the bundle $E$ (see $\S 6$ ). Vice versa, given a representation of $\tilde{\mathcal{P}}(M)$ in the fiber groupoid of the bundle $E$, there exists a connection $\nabla$ from which this representation arises as the associated parallel transport (see [6-7] and $\S 6))$.

A deeper result is the following. As we have seen, given a bundle-connection pair $(E, \nabla)$ the parallel transport operator $P^{\nabla}$ produces a representation of the loop group $\widetilde{\Omega}_{m}(M)$ on the fiber $E_{m}$. Conversely,

Translated from Matematicheskie Zametki, Vol. 61, No. 4, pp. 503-518, April, 1997.

Original article submitted September 23, 1995. 
a theorem stated by Kobayashi [8] in 1954 (proved in slightly different forms by Telemann in 1960 [914] and by Driver in 1989 [15]) says that it is possible to reconstruct a bundle-connection pair from a representation $P$ of the group $\widetilde{\Omega}_{m}(M)$. A similar result (not using the terminology of connections) has been proved by Lashof [16]. Note that the one-to-one correspondence between flat connections and representations of $\pi_{1}(M)$ and the equivalence of line bundle-connection pairs given by Kostant [17] based on parallel transport appear as particular cases of this theorem. An informal version of the theorem can be found in the physical literature in the paper of Giles [18]. I refer the reader to [18-24] for the meaning and applications of the theorem in gauge theory.

The purpose of the present paper is to put the proof of the theorem in a new and more suitable setting. We show that the algebraic structure of the proof can be greatly clarified if one looks at

$M=\widetilde{\mathcal{P}}_{m}(M) / \widetilde{\Omega}_{m}(M)$ as a homogeneous space for the action of the groupoid $\widetilde{\mathcal{P}}(M)$. In this way the reconstruction theorem of the bundle-parallel transport pair appears as a particular case of a reconstruction theorem that belongs to the theory of induced representations (when this last theory is suitably generalized to groupoids). It is possible to find papers describing the "algebraic" structure of the notion of connection (see [25]). Nevertheless the relationship between the main result of this note and the theory of induced representation appears to be entirely new. One should also note that a further relationship between the theory of connections and the theory of induced representations was already established in the physical and mathematical literature ([26-32]).

The structure of the paper is the following. In $\S \S 1-4$ we present the abstract theory of induced representation for groupoids: no topology is involved. In $\$ \S 5-6$ we discuss some known facts about manifolds of paths, connections and parallel transport. Section 7 contains the main result of the paper. In $\S 8$ we make some remarks about possible extensions of the main result to infinite-dimensional bundles.

\section{§1. Groupoids}

A groupoid $\mathcal{G}$ is a small category in which every morphism is an isomorphism. Intuitively, a groupoid is a "group" whose composition law is not everywhere defined. We write $\mathcal{G}=(M, \Gamma)$ to mean that $M$ is the set of objects (points) of $\mathcal{G}$ and $\Gamma$ is the set of morphisms (arrows) of $\mathcal{G}$. We denote by $\Gamma_{x, y}$ the set of isomorphisms between $x, y \in M$. If $\delta \in \Gamma_{x, y}$, we write $x=i(\delta)=$ initial point of $\delta$ and $y=f(\delta)=$ final point of $\delta$. We write also $\operatorname{Loop}(x):=\Gamma_{x, x}$. Obviously $\operatorname{Loop}(x)$ is a group. If $\Gamma_{x, y}=\varnothing$ for all $x, y \in M$, then $\mathcal{G}$ reduces to the set $M$ without any structure. If $M$ consists of a single object $x$, then $\mathcal{G}$ can be identified with the group Loop $(x)$ (obviously any group can be described in this way).

Definition 1.1. A groupoid homomorphism between $(M, \Gamma),\left(M^{\prime}, \Gamma^{\prime}\right)$ is a covariant functor $\psi$ : $(M, \Gamma) \rightarrow\left(M^{\prime}, \Gamma^{\prime}\right)$.

\section{§2. Actions of groupoids}

Let $X$ be a set. By $\mathcal{S}_{X}$ we denote the symmetric group over $X$, that is $\mathcal{S}_{X}:=\{f: X \rightarrow X \mid$ $f$ is bijective . If $G$ is a group, then an action of $G$ on $X$ is given by a homomorphism $L$ of $G$ into $S_{X}$. In this case we shall say also that $L$ is a representation of $G$ on $X$. We shall use action and representation as interchangeable terms. Let us generalize this notion to groupoids. If $X$ is a set, then we shall denote by $P(X)$ the power set of $X$, that is the set of all subsets of $X$. Suppose $A, B \subset X$. Define

$$
\Gamma_{A, B}:=\{f: A \rightarrow B \mid f \text { is bijective }\} \text { and } \Gamma(X)=\left\{\Gamma_{A, B} \mid A, B \subset X\right\} \text {. }
$$

Definition 2.1. Let $X$ be a set. $\mathcal{S}_{X, X}$ is the groupoid defined by $\mathcal{S}_{X, X}:=(P(X), \Gamma(X))$.

Note that $\mathcal{S}_{X}$ is a subgroupoid of $\mathcal{S}_{X, X}$ and, more precisely, $\mathcal{S}_{X}=$ Loop $(X)$.

Definition 2.2. Let $X$ be a set and let $\mathcal{G}=(M, \Gamma)$ be a groupoid, and suppose that a morphism $L: \mathcal{G} \rightarrow \mathcal{S}_{X, X}$ is given. We shall say equivalently that:

i) $\mathcal{G}$ acts on $X$;

ii) $X$ is a $\mathcal{G}$-space;

iii) there is a $\mathcal{G}$-action on $X$;

iv) $L$ is a representation of $\mathcal{G}$ on $X$. 
The action will be also denoted by the pair $(\mathrm{L}, \mathrm{X})$.

Note that this is a straightforward generalization of the definition given for groups. Suppose that an action $L: \mathcal{G}=(M, \Gamma) \rightarrow \mathcal{S}_{X, X}$ is given. For $\delta \in \Gamma$ we define

$$
\operatorname{Dom}_{X} \delta:=\operatorname{Dom}_{X} L_{\delta}:=i(L(\delta)), \quad \operatorname{Im}_{X} \delta:=\operatorname{Im}_{X} L_{\delta}:=f(L(\delta))
$$

that is $\operatorname{Dom}_{X} \delta, \operatorname{Im}_{X} \delta$ are respectively the domain and the image of $\delta$ (or $L_{\delta}$ ) when one looks at $\delta$ as a function defined on a subset of $X$.

Remark 2.1. Similarly to the group case, sometimes we simply write $\delta x=L_{\delta} x$ to denote the action of $\delta \in \mathcal{G}$ on $x \in X$ (so $\delta x$ is an element of $X$ ). In what follows, $\mathcal{G}$ is a groupoid and $X$ is a $\mathcal{G}$-space.

Definition 2.3. Let $x \in X$. We define two subgroupoids of $\mathcal{G}$ (simply specifying the sets of arrows)

$$
\mathcal{G}_{x}:=\left\{\delta \in \mathcal{G} \mid x \in \operatorname{Dom}_{X} \delta\right\} \quad \mathcal{G}_{x, y}:=\left\{\delta \in \mathcal{G}_{x} \mid \delta x=y\right\} \quad \Omega_{x}:=\mathcal{G}_{x, x}
$$

Definition 2.4. We say that $\mathcal{G}$ acts transitively on $X$ if for all $x, y \in X$ we have $\mathcal{G}_{x, y} \neq \varnothing$.

Proposition 2.1. Let $X$ be a transitive $\mathcal{G}$-space. Fix $x \in X$. Then $\Omega_{x}$ is a subgroup acting naturally by right multiplication on the subgroupoid $\mathcal{G}_{x}$. We have an identification of $X$ with the quotient set $\mathcal{G}_{x} / \Omega_{x}$.

Proof. The same as in the group case.

Definition 2.5. A subgroupoid $\Omega \subseteq \mathcal{G}$ is an isotropy subgroup if there exists a transitive $\mathcal{G}$-space $X$ and an element $x \in X$ such that $\Omega=\bar{\Omega}_{x}$.

\section{§3. $\mathcal{G}$-structures}

Definition 3.1. Let $\mathcal{G}$ be a groupoid. A triple $(A, B, \pi)$ is a $\mathcal{G}$-structure if

i) $A$ is a $\mathcal{G}$-space;

ii) $B$ is a transitive $\mathcal{G}$-space;

iii) $\pi: A \rightarrow B$ is a surjective map such that for all $x \in B$, for all $\delta \in \mathcal{G}$

$$
\begin{gathered}
x \in \operatorname{Dom}_{B} \delta \Rightarrow A_{x}:=\pi^{-1}(x) \subset \operatorname{Dom}_{A} \delta \\
\delta A_{x}=A_{\delta x} .
\end{gathered}
$$

Example 3.1. Construction of $\mathcal{G}$-structures.

Suppose $\mathcal{G}$ is a groupoid, $\Omega$ is an isotropy subgroup of $\mathcal{G}$ and $L$ is a representation of $\Omega$ on the space $V$. By definition of the isotropy subgroup, there exist a $\mathcal{G}_{\text {-set }} B$ and $x \in B$ such that $B \cong \mathcal{G}_{x} / \Omega_{x}$, $\Omega=\Omega_{x}$. We can make $\mathcal{G}_{x} \times V$ into an $\Omega_{x}$-space by defining

$$
(\sigma, v) \gamma:=\left(\sigma \gamma, \gamma^{-1} v\right)=\left(\sigma \gamma, L_{\gamma^{-1}} v\right) \quad \sigma \in \mathcal{G}_{x}, \gamma \in \Omega_{x}, v \in V
$$

We denote by $[(\sigma, v)] \in A^{L}:=\mathcal{G}_{x} \times{ }_{L} V:=\mathcal{G}_{x} \times V / \Omega_{x}$ the equivalence class of $(\sigma, v)$ in $\mathcal{G}_{x} \times V$ and by $[\sigma] \in \mathcal{G}_{x} / \Omega_{x}$ the equivalence class of $\sigma$ in $\mathcal{G}_{x}$. Set $\pi[(\sigma, v)]:=[\sigma]$.

Definition 3.2. The associated action $\left(R^{L}, A^{L}\right)$ of $\mathcal{G}$ is defined by (whenever this makes sense)

$$
R_{\delta}^{L}[(\sigma, v)]:=\delta[(\sigma, v)]:=[(\delta \sigma, v)] \quad \delta \in \mathcal{G}, \sigma \in \mathcal{G}_{x}, v \in V .
$$

Remark 3.1. If one considers $\mathcal{G}_{x}$ as a "principal $\Omega_{x}$-bundle" over $X=\mathcal{G}_{x} / \Omega_{x}$, then $A^{L}:=\mathcal{G}_{x} \times{ }_{L} V$ is the "associated bundle" and this explains the terminology.

Notice that the action of $\mathcal{G}$ on $B \simeq \mathcal{G}_{x} / \Omega_{x}$ is given by (whenever this makes sense) $\delta[\sigma]=[\delta \sigma]$. 
Remark 3.2. Let $y \in B$, then $y=[\sigma]$ is equivalent to $\sigma x=y$ and therefore,

$$
\delta[\sigma]=\delta y=\delta \sigma x=[\delta \sigma] .
$$

Moreover, if $y \in \operatorname{Dom}_{B} \delta$ (that is, $\delta \sigma$ makes sense), then

$$
[(\tilde{\sigma}, v)] \in A_{y}^{L}=\pi^{-1}(y) \Rightarrow[\tilde{\sigma}]=[\sigma] \Rightarrow \tilde{\sigma}=\sigma \gamma \quad \gamma \in \Omega_{x} \Rightarrow \delta \tilde{\sigma}=\delta \sigma \gamma \text { makes sense. }
$$

Therefore, we have defined a $\mathcal{G}$-structure

$$
\begin{array}{ccc}
A^{L} & = & \mathcal{G}_{x} \times{ }_{L} V \\
\pi \downarrow & \downarrow \pi \\
B & =\mathcal{G}_{x} / \Omega_{x}
\end{array}
$$

Remark 3.3. In view of Driver's suggestion for the proof of Theorem 7.1 [22], we note the following. Let $\tau \in \mathcal{G}_{x}$ be such that $[\tau]=[\delta \sigma]$. This is equivalent to $\left(\tau^{-1} \delta \sigma\right) \in \Omega_{x}$. Therefore,

$$
\left[\left(\tau,\left(\tau^{-1} \delta \sigma\right) v\right)\right]=\left[\left(\tau\left(\tau^{-1} \delta \sigma\right),\left(\tau^{-1} \delta \sigma\right)^{-1}\left(\tau^{-1} \delta \sigma\right) v\right)\right]=[(\delta \sigma, v)]=\delta[(\sigma, v)]
$$

and it is possible to define the associated representation by

$$
R_{\delta}^{L}[(\sigma, v)]:=\delta[(\sigma, v)]:=\left[\left(\tau,\left(\tau^{-1} \delta \sigma\right) v\right)\right],
$$

where $\tau$ is an arbitrary element with the property $\left(\tau^{-1} \delta \sigma\right) \in \Omega_{x}$.

Definition 3.3. Any $\mathcal{G}$-structure of the form $\left(\mathcal{G}_{x} \times{ }_{L} V, \mathcal{G}_{x} / \Omega_{x}, \pi\right)$ is called canonical.

Definition 3.4. An isomorphism between two $\mathcal{G}$-structure $(A, B, \pi),\left(A^{\prime}, B^{\prime}, \pi^{\prime}\right)$ is given by a pair of bijective functions $\varphi: A \rightarrow A^{\prime}, \psi: B \rightarrow B^{\prime}$ such that

i) $\pi^{\prime}(\varphi(a))=\psi(\pi(a))$

ii) $\varphi(\delta a)=\delta \varphi(a)$

iii) $\psi(\delta x)=\delta \psi(x)$.

Proposition 3.1. Any $\mathcal{G}$-structure $(A, B, \pi)$ is isomorphic to a canonical one.

Proof. Fix $x \in B$. Consider the isotropy subgroup $\Omega_{x}$. Obviously, $A_{x}$ is an $\Omega_{x}$-space. We may construct the canonical $\mathcal{G}$-structure $\left(\mathcal{G}_{x} \times A_{x} / \Omega_{x}, \mathcal{G}_{x} / \Omega_{x}, \pi^{\prime}\right)$. Define

$$
\left.\varphi: \mathcal{G}_{x} \times A_{x} / \Omega_{x} \rightarrow A \quad \text { by } \varphi([\sigma, v)]\right)=\sigma v \quad \psi: \mathcal{G}_{x} / \Omega_{x} \rightarrow B \quad \text { by } \psi([\sigma])=\sigma x .
$$

It is easy to see that the pair $(\varphi, \psi)$ is an isomorphism.

Remark 3.4. We may rephrase the content of this proposition by saying that a $\mathcal{G}$-structure is entirely encoded in the action of the isotropy subgroup (of a fixed point $x$ ) over the fiber (that is, over $\pi^{-1}(x)$ ).

Example 3.2. Let $G$ be an arbitrary group. Any subgroup $H$ is obviously an isotropy subgroup (consider the canonical transitive action of $G$ on $G / H$ ). Therefore, given a representation $L$ of $H$ on a space $V$, we may construct the $G$-structure $\left(A^{L}, B, \pi\right)=\left(G \times{ }_{L} V, G / H, \pi\right)$.

Example 3.3. Let $(E, M, \pi)$ be a bundle over a connected differentiable manifold $M$. Let $\widetilde{\mathcal{P}}(M)$ be the groupoid of paths on this manifold. $\widetilde{\mathcal{P}}(M)$ acts naturally on $M$ if we mean that $\delta \in \widetilde{\mathcal{P}}(M)$ takes its initial point $x=i(\delta)$ to $y=f(\delta)$, the final point. The isotropy group of $m \in M$ is exactly the loop group $\widetilde{\Omega}_{m}(M)$. We have also the groupoid $\widetilde{\mathcal{P}}_{m}(M)$ of paths starting from $m$ and a natural identification $\widetilde{\mathcal{P}}_{m}(M) / \widetilde{\Omega}_{m}(M) \cong M$.

To say that a parallel transport over $(E, M, \pi)$ is given is simply to say (see Definition 6.3 ) that $(E, M, \pi)$ is a $\tilde{\mathcal{P}}(M)$-structure with the action of $\tilde{\mathcal{P}}(M)$ on $M$ given as above. Proposition 3.1 shows that every such $\widetilde{\mathcal{P}}(M)$-structure $(E, M, \pi)$ can be identified with a $\widetilde{\mathcal{P}}(M)$-structure

$$
\left(\mathcal{P}_{m}(M) \times E_{m} / \Omega_{m}(M) ; \mathcal{P}_{m}(M) / \Omega_{m}(M), \pi\right)
$$

given by a representation of $\widetilde{\Omega}_{m}(M)$. 


\section{$\S 4$. Induced representations for groupoids}

Let $\Omega=\Omega_{x}$ be an isotropy group of the groupoid $\mathcal{G}$. Suppose an $\Omega$-action $L$ is given on the space $V$. Let $\left(A^{L}, B, \pi\right)=\left(\mathcal{G}_{x} \times_{L} V, \mathcal{G}_{x} / \Omega_{x}, \pi\right)$ be the corresponding $\mathcal{G}$-structure. Let $X \subset B$. We define

$$
\mathcal{S}\left(X, A^{L}\right)=\left\{f: X \rightarrow A \mid \pi \circ f=\operatorname{Id}_{X}\right\} .
$$

$\mathcal{S}\left(X, A^{L}\right)$ is the set of sections on $X$.

Definition 4.1.

$$
V^{L}:=\bigcup_{\gamma \in \mathcal{G}} \mathcal{S}\left(\operatorname{Dom}_{B} \gamma, A^{L}\right)
$$

We shall define on $V^{L}$ a new representation $U^{L}$ of $\mathcal{G}$ that will be called the induced representation of $\mathcal{G}$.

Remark 4.1. There are two extreme cases.

1) If $\operatorname{Dom}_{B} \gamma$ reduces to the singleton $\{x\}$ (as in the case $\mathcal{G}=\tilde{\mathcal{P}}(M), \Omega=\widetilde{\Omega}_{m}(M)$ ), then, with a little abuse of language, we can write

$$
\mathcal{S}\left(\operatorname{Dom}_{B} \gamma, A^{L}\right)=\mathcal{S}\left(x, A^{L}\right)=A_{x}^{L}
$$

and therefore,

$$
V^{L}:=\bigcup_{\gamma \in \mathcal{G}} \mathcal{S}\left(\operatorname{Dom}_{B} \gamma, A^{L}\right)=\bigcup_{x \in B} A_{x}^{L}=A^{L} .
$$

2) If $\operatorname{Dom}_{B} \gamma=B$ for all $\gamma \in \mathcal{G}$ (as in the case $\mathcal{G}=G=$ group and $\Omega=H=$ subgroup), then

$$
V^{L}=\bigcup_{\gamma \in \mathcal{G}} \mathcal{S}\left(\operatorname{Dom}_{B} \gamma, A^{L}\right)=\bigcup_{\gamma \in \mathcal{G}} \mathcal{S}\left(B, A^{L}\right)=\mathcal{S}\left(B, A^{L}\right) .
$$

Definition 4.2. Let $f \in \mathcal{S}\left(\operatorname{Dom}_{B} \gamma, A^{L}\right)$. The induced representation $\left(U^{L}, V^{L}\right)$ is defined by

$$
\left(U_{\gamma}^{L} f\right)(y):=\gamma f\left(\gamma^{-1} y\right) \quad \text { where } \quad y \in \operatorname{Dom}_{B} \gamma^{-1} \text {. }
$$

From the definition we have

$$
U_{\gamma}^{L}: \mathcal{S}\left(\operatorname{Dom}_{B} \gamma, A^{L}\right) \rightarrow \mathcal{S}\left(\operatorname{Dom}_{B} \gamma^{-1}, A^{L}\right)
$$

Remark 4.2. In the case $\mathcal{G}=G=$ group, $\Omega=\Omega_{x}=H=$ subgroup we have the usual definition of induced representation (action).

Proposition 4.1. Let $\left(A^{L}, B, \pi\right)=\left(\mathcal{G}_{x} \times_{L} V, \mathcal{G}_{x} / \Omega_{x}, \pi\right)$ be a $\mathcal{G}$-structure such that

$$
\operatorname{Dom}_{B} \gamma=\{x\}=\text { singleton } \quad \forall \gamma \in \mathcal{G} \text {. }
$$

Then the associated representation $\left(R^{L}, A^{L}\right)$ of $\mathcal{G}$ coincides with the induced representation $\left(U^{L}, V^{L}\right)$, that is

$$
U_{\delta}^{L}[(\sigma, v)]=[(\delta \sigma, v)]=R_{\delta}^{L}[(\sigma, v)]
$$

Proof. First of all it follows from Remark 4.1 that $V^{L}=A^{L}$, where $a=[(\sigma, v)] \in A_{x}^{L}$ is identified with the function $f_{a}(\cdot):\{x\} \rightarrow A_{x}^{L}\left(f_{a}(\cdot) \in \mathcal{S}(x, A)\right)$ such that $f_{a}(x)=a$. Let $\delta x=y=\operatorname{Dom}_{B} \delta^{-1}$ so that $x=\delta^{-1} y$. We have

$$
U_{\delta}^{L}[(\sigma, v)]=U_{\delta}^{L} a=\left(U_{\delta}^{L} f_{a}\right)(y)=\delta f_{a}\left(\delta^{-1} y\right)=\delta f_{a}(x)=\delta a=\delta[(\sigma, v)]=R_{\delta}^{L}[(\sigma, v)]
$$

and the required assertion. 
Remark 4.3. Note that in the case $\mathcal{G}=G=$ group the conclusion of Proposition 4.1 does not hold.

Remark 4.4. The assumption of Proposition 4.1 applies to the case of Example 3.3, since for any curve $\gamma \in \widetilde{\mathcal{P}}(M)$ the domain $i(\gamma)$ is a singleton.

Putting together Propositions 3.1 and 4.1, we have bijective correspondences between

- representations $L$ of $\widetilde{\Omega}_{m}(M)$;

- $\widetilde{\mathcal{P}}(M)$-structures on bundles $(E, M, \pi)$ (that is, parallel transports);

- representations $U^{L}$ of $\widetilde{\mathcal{P}}(M)$ induced by representations $L$ of $\widetilde{\Omega}_{m}(M)$.

This is the "algebraic" content of the main result (Theorem 7.1) of the present paper. With little abuses of language one may rephrase the above remark in several ways. For example, one may say that

- A connection $\nabla$ is a representation $P^{\nabla}=U^{L}$ of $\tilde{\mathcal{P}}(M)$ induced by a representation $L$ of $\widetilde{\Omega}_{m}(M)$;

- A representation $P$ of $\tilde{\mathcal{P}}(M)$ is equal to the parallel transport $P^{\nabla}$ of a connection $\nabla$ if and only if it is induced by a representation $L$ of $\widetilde{\Omega}_{m}(M)$.

\section{§5. Manifolds and groupoids of paths}

In this section we closely follow [15]. Let $(V,(\cdot, \cdot))$ be a finite-dimensional inner product space, and let $J$ be a subinterval of $I \equiv[0,1]$. Define

$$
\|\sigma\|_{1}^{2}=\int_{J}\left(\left|\sigma^{\prime}(t)\right|^{2}+|\sigma(t)|^{2}\right) d t
$$

Let $A C$ stand for absolutely continuous. Put $H^{1}(J, V):=\left\{\sigma \in A C(J, V)\|\| \sigma \|_{1}<+\infty\right\} . H^{1}(J, V)$ is an infinite-dimensional Hilbert space. Let $M$ be a fixed $n$-dimensional manifold. A path $\sigma: J \rightarrow M$ will be called $H^{1}$ if for each chart $\left(\varphi_{\alpha}, U_{\alpha}\right)$ and for each subinterval $K \subset J$ such that $\sigma(K) \subset U_{\alpha}$ the function $\left.\varphi_{\alpha} \circ \sigma\right|_{K}: K \rightarrow \mathbb{R}^{n}$ is contained in $H^{1}\left(K, \mathbb{R}^{n}\right)$ (this notion is chart independent). We shall denote by $\mathcal{P}(M):=\mathcal{P}^{1}(M):=H^{1}(I, M)$ the set of $H^{1}$ curves $\sigma: I \rightarrow M$. We also define

$$
\mathcal{P}_{m}(M):=\{\sigma \in \mathcal{P}(M) \mid \sigma(0)=m \in M\}, \quad \Omega_{m}(M):=\left\{\sigma \in \mathcal{P}_{m}(M) \mid \sigma(1)=m\right\}
$$

It is possible to prove the following theorem.

Theorem 5.1. $\mathcal{P}(M)$ is a Hilbert manifold modeled on $H^{1}(I, \mathbb{R})$. Then $\mathcal{P}_{m}(M)$ and $\Omega_{m}(M)$ are submanifolds of $\mathcal{P}(M)$.

Proof. See [15].

Define $\mathcal{R}(I):=\left\{\mathcal{C}^{1}\right.$-diffeomorphisms of $I$ such that $\left.r(0)=0, r(1)=1\right\}$. On $\mathcal{P}(M)$ let us define two admissible operations:

i) $\sigma \rightarrow \sigma \circ r$ where $r \in \mathcal{R}(I)$ (reparametrization);

ii) $\sigma \cdot \beta \cdot \beta^{-1} \gamma \rightarrow \sigma \cdot \gamma$, where $\beta^{-1}$ is the curve $\beta$ passed in the opposite direction (cutting of appendices).

Definition 5.1. We say that $\sigma \sim \widehat{\sigma}$ in $\mathcal{P}(M)$ if $\widehat{\sigma}$ can be obtained from $\sigma$ by performing a finite sequence of admissible operations i), ii).

Of course, $\sim$ is an equivalence relation on $\mathcal{P}(M)$. Put

$$
\widetilde{\mathcal{P}}(M):=\mathcal{P}(M) / \sim, \quad \widetilde{\mathcal{P}}_{m}(M):=\tilde{\mathcal{P}}_{m}(M) / \sim, \quad \widetilde{\Omega}_{m}(M):=\Omega_{m}(M) / \sim .
$$

For $\sigma \in \mathcal{P}(M)$ we shall indicate by $\tilde{\sigma}$ the equivalence class of $\sigma$.

Proposition 5.1. $\widetilde{\mathcal{P}}(M), \widetilde{\mathcal{P}}_{m}(M), \widetilde{\Omega}_{m}(M)$ have a groupoid (group) structure with respect to the operation given by the concatenation of (unparameterized) curves. 


\section{Proof. Obvious.}

Suppose we are given a function $P: \mathcal{P}(M) \rightarrow X$ such that $P$ is parameterization invariant, that is

$$
P(\sigma \circ r)=P(\sigma) \quad \forall r \in \mathcal{R}(I)
$$

and appendix invariant, that is

$$
P\left(\sigma \cdot \beta \cdot \beta^{-1} \tau\right)=P(\sigma \cdot \tau) \quad \forall \beta \in \mathcal{P}(M)
$$

In this case we may define $\widetilde{P}: \widetilde{\mathcal{P}}(M) \rightarrow X$ by $\widetilde{P}(\widetilde{\sigma}):=P(\sigma)$. Vice versa, given a function $\widetilde{P}: \tilde{\mathcal{P}}(M) \rightarrow X$ we get a parameterization and appendix invariant (p.a.i.) function $P: \mathcal{P}(M) \rightarrow X$ defined by

$$
P(\sigma):=\widetilde{P}(\widetilde{\sigma})
$$

The typical example of such a function will be the parallel transport. Suppose we have a function $\widetilde{P}$ : $\widetilde{\mathcal{P}}(M) \rightarrow N$ where $N$ is a smooth manifold. By abuse of language we shall say that $\widetilde{P}$ is $C^{k}$-differentiable if the associated p.a.i. function $P: \mathcal{P}(M) \rightarrow N$ is $C^{k}$-differentiable.

\section{§6. Vector bundles, linear connections and parallel transports}

Suppose $E, M$ are smooth finite-dimensional manifolds, $V$ is a complex or real finite-dimensional vector space, $G$ is a closed subgroup of $\mathrm{GL}(V)$. If

i) there is a surjective smooth function $\pi: E \rightarrow M$ such that $E_{x}:=\pi^{-1}(x)$ is a vector space isomorphic to $V$ for all $x \in M$;

ii) $M$ has an atlas $\left\{U_{\alpha}\right\}_{\alpha \in \mathcal{A}}$ such that for any $\alpha$ there is a bijective map $\varphi_{\alpha}: \pi^{-1}\left(U_{\alpha}\right) \rightarrow U_{\alpha} \times V$ for which the following diagram is commutative

$$
\begin{array}{ccc}
\pi^{-1}\left(U_{\alpha}\right) & \stackrel{\varphi_{\alpha}}{\longrightarrow} & U_{\alpha} \times V \\
\pi \downarrow & & \downarrow \\
U_{\alpha} & \longrightarrow & U_{\alpha}
\end{array}
$$

iii) for any $U_{\alpha}, U_{\beta}$ (charts of the atlas) the functions $G \ni g_{\alpha \beta}(x):=\varphi_{\alpha} \circ \varphi_{\beta}^{-1}(x, \cdot): V \rightarrow V$, are smooth for all $x \in U_{\alpha} \cap U_{\beta}$;

then we say that $E=(E, \pi, M, V, G)$ is a vector bundle over $M$ with typical fiber $V$, structure group $G$, and transition functions $g_{\alpha \beta}$.

The transition functions $g_{\alpha \beta}(\cdot)$ of a vector bundle $E$ satisfy the cocycle condition

$$
g_{\alpha \beta}(x) g_{\beta \gamma}(x)=g_{\alpha \gamma}(x) \quad \forall x \in U_{\alpha} \cap U_{\beta} \cap U_{\gamma}
$$

Proposition 6.1. Given a manifold $M$, a vector space, a closed subgroup $G \subset \mathrm{GL}(V)$ and a family of functions $\left\{g_{\alpha \beta}(\cdot)\right\}$ satisfying relations $(*)$ (relatively to a cover $\left\{U_{\alpha}\right\}_{\alpha \in \mathcal{A}}$ of $M$ ), there exists a bundle $E=(E, \pi, M, V, G)$ whose transition functions are the $g_{\alpha \beta}$.

Proof. See [33].

We shall denote by $S(E)$ the $\mathcal{C}^{\infty}(M)$-module of smooth sections of the bundle $E$. Therefore, $S(T M)$ is the module of vector fields of the manifold.

Definition 6.1. A linear connection (or a covariant derivative) on $E$ is a bilinear function

$$
\nabla: S(T M) \times S(E) \rightarrow S(E), \quad(v, s) \mapsto \nabla_{v} s
$$

such that for all $f \in \mathcal{C}^{\infty}(M)$

1) $\nabla_{f v} s=f \nabla_{v} s$

2) $\nabla_{v}(f s)=f \nabla_{v} s+(v f) s$.

In what follows, we shall consider only covariant derivatives compatible with the structure group $G$ (see [15-22]). Now we introduce the notion of parallel transport on $E=(E, \pi, M, V, G)$. 
Definition 6.2. The fiber groupoid $\tau(E)$ of a bundle $E$ is the groupoid whose points are given by the fibers $\left\{E_{x}\right\}_{x \in M}$ and whose arrows are the linear isomorphisms $\left\{\mathrm{GL}\left(E_{x}, E_{y}\right)\right\}$. Note that the set of points of $\tau(E)$ can be identified with $M$.

Definition 6.3. A linear parallel transport over $E$ is a homomorphism $P: \tilde{\mathcal{P}}(M) \rightarrow \tau(E)$ respecting the natural projections of $\widetilde{\mathcal{P}}(M), \tau(E)$ on $M$.

A linear parallel transport over $E$ is compatible with the structure group $G$ if the following properties hold.

i) Let $P(\sigma)_{\alpha \beta}:=\varphi_{\alpha}(y, \cdot) P(\sigma) \varphi_{\beta}^{-1}(x, \cdot): V \rightarrow V \quad y \in U_{\alpha}, x \in U_{\beta}$. Then $P(\sigma)_{\alpha \beta} \in G$ for any local trivialization $\alpha, \beta \in \mathcal{A}$.

ii) Let $\left(\varphi_{\alpha}, U_{\alpha}\right)$ be a local trivialization and let $\sigma, \sigma^{\prime}$ be curves in $U_{\alpha}$ starting at $x_{0}$ with the same tangent vector $X \in T_{x_{0}}(M)$. Then

$$
G \ni P(\sigma(t))_{\alpha \alpha}: \varphi_{\alpha}(\sigma(t), \cdot) P(\sigma(t)) \varphi_{\alpha}^{-1}(\sigma(0), \cdot): V \rightarrow V
$$

Therefore, $P(\sigma(\cdot))_{\alpha \alpha}, P\left(\sigma^{\prime}(\cdot)\right)_{\alpha \alpha}$ are curves in $G$ starting at the identity. We require that the curves $P(\sigma(\cdot))_{\alpha \alpha}, P\left(\sigma^{\prime}(\cdot)\right)_{\alpha \alpha}$ have the same tangent vector $\theta_{\alpha}\left(x_{0}, X\right)$ depending smoothly (and linearly) on $x_{0}$ and on $X$.

In what follows, we consider only linear parallel transports compatible with the structure group $G$.

Let $\sigma \in \mathcal{P}(M)$, i.e., $\sigma:[0,1] \rightarrow M$; we define

$$
\sigma^{s}:[0,1] \rightarrow M \quad \text { by } \sigma^{s}(t):=\sigma(s t) \text { for all } t \in[0,1] \text {. }
$$

Obviously, $\sigma^{s} \in \mathcal{P}(M)$ for all $s \in[0,1]$ and $\sigma^{s}(0)=\sigma(0), \sigma^{s}(1)=\sigma(s)$. We use this notation in the following theorem.

Theorem 6.1. There is a one-to-one correspondence between parallel transports and covariant derivatives over a bundle $E$.

Proof. Suppose we have a parallel transport $P$. We may define a covariant derivative $\nabla^{P}$ in the following way. Let $x \in M$, and let $\sigma:[0,1] \rightarrow M$ be a smooth curve such that $x=\sigma(0)$. We put for a section $s \in S(E)$

$$
\left(\nabla_{\dot{\sigma}(0)}^{P} s\right)(x):=\lim _{h \rightarrow 0} \frac{1}{h}\left[P\left(\sigma^{h}\right)^{-1} s(\sigma(h))-s(x)\right] .
$$

If $v \in T_{x} M$ and $\sigma(0)=x, \dot{\sigma}(0)=v$, then it is easy to see that

$$
\left(\nabla_{v}^{P} s\right)(x):=\left(\nabla_{\dot{\sigma}(0)}^{P} s\right)(x)
$$

is independent of the chosen curve $\sigma(\cdot)$ (i.e., that $\left(\nabla_{v}^{P} s\right)(x)$ is well defined).

For a vector field $V \in S(T M)$ we put

$$
\left(\nabla_{V}^{P} s\right)(x):=\left(\nabla_{V(x)}^{P} s\right)(x)
$$

It is well known that $\nabla^{P}$ has the defining properties of a covariant derivative (see [34]). Conversely, given a covariant derivative $\nabla$ on $E$, we may define a parallel transport $P^{\nabla}$ in the following way.

Let $\sigma:[0,1] \rightarrow M$ be a smooth curve. The following first order ODE admits the unique solution, $v(\cdot)$.

$$
\nabla_{\dot{\sigma}(t)} v(\sigma(t))=0 \quad v(\sigma(0))=v_{0} \in E_{\sigma(0)}=\pi^{-1}(\sigma(0))
$$

Define $P^{\nabla}(\sigma) v_{0}:=v(1)$. Varying $v_{0}$, we get a linear isomorphism $P^{\nabla}(\sigma): E_{\sigma(0)} \rightarrow E_{\sigma(1)}$ satisfying the defining properties of a parallel transport. It is easy to see that $\nabla^{P^{\nabla}}=\nabla$ and $P^{\nabla^{P}}=P$, and this concludes the proof. 


\section{$\S 7$. From loop group representations to parallel transports}

Definition 7.1. A bundle-connection pair $(E, \nabla)$ is consists of a vector bundle $E=(E, \pi, M, V, G)$ and a linear connection $\nabla$ on $E$. Two pairs $(E, \nabla),\left(E^{\prime}, \nabla^{\prime}\right)$ are said to be equivalent if there is a vector bundle isomorphism $\varphi: E \rightarrow E^{\prime}$ such that

1) $\varphi \nabla=\nabla^{\prime} \varphi$

2) $\varphi$ respects the structure group (see [15]).

By $[E, \nabla]$ we shall denote the equivalence class containing $(E, \nabla)$. We write $\mathcal{E}=\mathcal{E}(M, V, G)$ for the set of all these equivalence classes.

Definition 7.2. A bundle-parallel transport pair $(E, P)$ is a pair consisting of a vector bundle $E=$ $(E, \pi, M, V, G)$ and a linear parallel transport $P$ on $E$. Two pairs $(E, P),\left(E^{\prime}, P^{\prime}\right)$ are said to be equivalent if there is a vector bundle isomorphism $\varphi: E \rightarrow E^{\prime}$ such that

1) $\varphi P=P^{\prime} \varphi$;

2) $\varphi$ respects the structure group.

By $[E, P]$ we shall denote the equivalence class containing $(E, \nabla)$. We write $\mathcal{T}=\mathcal{T}(M, V, G)$ for the set of all these equivalence classes.

Proposition 7.1. There is bijective a correspondence between $\mathcal{E}$ and $\mathcal{T}$ given by the parallel transport operator

$$
[E, \nabla] \rightarrow\left[E, P^{\nabla}\right] .
$$

Proof. Immediate consequence of Theorem 6.1.

Definition 7.3. Given a smooth representation $L: \widetilde{\Omega}_{m}(M) \rightarrow G \subset \mathrm{GL}(V)$ (see §5), we shall denote by $[L]$ the equivalence class of this representation with respect to conjugation in $G$. We shall write $\mathcal{R}=\mathcal{R}(M, m, V, G)$ for the set of all these equivalence classes.

Remark 7.1. Concerning the fact that a parallel transport provides a smooth representation of $\widetilde{\Omega}_{m}(M)$, see [15], Corollary 5.1, and [21], Theorem 2.2.

Now we may state the main result.

Theorem 7.1. There is a bijective correspondence between $\mathcal{E}$ and $\mathcal{R}$ given by the parallel transport operator

$$
[E, \nabla] \rightarrow\left[P^{\nabla}\right]
$$

Remark 7.2. Driver defines $\mathcal{M}=\mathcal{M}(M, m, V, G)$ as the class of SDPIM functions, that is of strongly differentiable, parameterization invariant, multiplicative functions $L: \Omega_{m}(M) \rightarrow G$. Theorem 7.1 of [15] states that $[E, \nabla] \rightarrow\left[P^{\nabla}\right]$ is a bijective correspondence between $\mathcal{E}$ and $\mathcal{M}$. Note that strongly differentiable means (by definition) smooth plus an analytic condition that is used (see Lemma 7.1 in [15]) to show that a SDPIM is appendix invariant. Therefore, a SDPIM determines an element of $\mathcal{R}$. Theorem 7.1 of the present paper establishes the opposite correspondence. Therefore, there is a bijective correspondence between the class $\mathcal{M}$ of SDPIM functions and the class $\mathcal{R}$ of smooth representations of $\widetilde{\Omega}_{m}(M)$.

Proof of Theorem 7.1. We simply prove here that given a smooth representation $L: \widetilde{\Omega}_{m}(M) \rightarrow G$, there exists a $\left[E, P^{\nabla}\right] \in \mathcal{T}$ such that $\left[P^{\nabla}\right]=[L]$ (see [15] for the missing part). We divide the proof in three steps.

Step 1: Reconstruction of the bundle-parallel transport pair (see Examples 3.1-3.3, Proposition 4.1, Remark 4.4).

Given the representation $L$ of $\widetilde{\Omega}_{m}(M)$ on the space $V$, we may consider the induced representation $U^{L}$ of $\tilde{\mathcal{P}}(M)$ on the space $V^{L}$. Define $[E, P]=\left[V^{L}, U^{L}\right]$. Remember that in this case the associated representation and the induced representation coincide so that

$$
E:=V^{L}:=\widetilde{\mathcal{P}}_{m}(M) \times_{L} V=\widetilde{\mathcal{P}}_{m}(M) \times V / \widetilde{\Omega}_{m}(M),
$$


where the action of $\sigma$ on $\widetilde{\mathcal{P}}_{\boldsymbol{m}}(M) \times V$ is given by

$$
(\sigma, v) \gamma:=\left(\sigma \gamma, L_{\left(\gamma^{-1}\right)} v\right)
$$

The projection $\pi: E \rightarrow M=\widetilde{\mathcal{P}}_{m}(M) / \widetilde{\Omega}_{m}(M)$ is given by $\pi[(\sigma, v)]=f(\sigma)$ ( $\pi$ is obviously well defined). For $[(\sigma, v)] \in E_{x}, \delta \in G$, and $x=f(\sigma)=i(\delta)$, we have

$$
P(\delta)[(\sigma, v)]:=U_{\delta}^{L}[(\sigma, v)]=R_{\delta}^{L}[(\sigma, v)]=\delta[(\sigma, v)]:=[(\delta \sigma, v)] .
$$

The representation $P=U^{L}$ of $\tilde{\mathcal{P}}(M)$ on $E=V^{L}$ defined above has all the algebraic properties of a parallel transport and, moreover, the parallel transport around loops based at $m$ coincides with the representation $L$ of $\widetilde{\Omega}_{m}(M)$ that we started with. This follows immediately from the considerations of $\S \S 3,4$.

Step 2: Local trivialization of the bundle.

Definition 7.4 (see [15]). Let $U$ be an open subset of $M$. Let $\mathrm{I}=[0,1]$. A smooth function $\Sigma$ : $I \times U \rightarrow M$ such that $\Sigma(1, x)=x$ and $\Sigma(0, x)=m$ for all $x \in U$ will be called an $m$-contraction over $U$ (or, simply, an $m$-contraction). Obviously, $\Sigma(\cdot, x)$ is a curve from $m$ to $x$.

Let us fix once and for all a collection $\left\{\left(\Sigma_{\alpha}, U_{\alpha}\right)\right\}$ of $m$-contractions over a fixed open cover $\left\{U_{\alpha}\right\}$ of $M$. We shall write also $\Sigma_{\alpha}^{x}:=\Sigma_{\alpha}^{x}(\cdot):=\Sigma_{\alpha}(\cdot, x)$. Let $\Sigma_{m}(M)$ be the set of the curves $\Sigma_{\alpha}^{x}$. Consider the following equivalence relation $\sim$ on $\Sigma_{m}(M) \times V$ :

$$
\left(\Sigma_{\alpha}^{x}, v\right) \sim\left(\Sigma_{\beta}^{y}, w\right) \quad \text { iff } \quad x=y \quad \text { and } \quad P\left(\Sigma_{\beta}^{x^{-1}} \Sigma_{\alpha}^{x}\right) v=w
$$

We have a projection $\pi:\left(\Sigma_{m}(M) \times V / \sim\right) \rightarrow M$ defined by $\pi\left[\left(\Sigma_{\beta}^{x}, v\right)\right]=x$.

Lemma 7.1. Define $g_{\alpha \beta}(x):=P\left(\Sigma_{\alpha}^{x-1} \Sigma_{\beta}^{x}\right)$. Then

i) The $\left\{g_{\alpha \beta}(x)\right\}$ form the transition functions of a vector bundle $F$;

ii) The bundle $F$ can be identified with $\pi:\left(\Sigma_{m}(M) \times V / \sim\right) \rightarrow M$.

Proof. To prove i) it is enough to show that the $g_{\alpha \beta}$ satisfy the cocycle condition

$$
g_{\alpha \beta}(x) g_{\beta \gamma}(x)=g_{\alpha \gamma}(x) \quad \text { for } \quad x \in U_{\alpha} \cap U_{\beta} \cap U_{\gamma}
$$

Since $P$ is a representation of $\widetilde{\Omega}_{m}(M)$, we have

$$
g_{\alpha \beta}(x) g_{\beta \gamma}(x)=P\left(\Sigma_{\alpha}^{x^{-1}} \Sigma_{\beta}^{x}\right) P\left(\Sigma_{\beta}^{x^{-1}} \Sigma_{\gamma}^{x}\right)=P\left(\Sigma_{\alpha}^{x^{-1}} \Sigma_{\beta}^{x} \Sigma_{\beta}^{x^{-1}} \Sigma_{\gamma}^{x}\right)=P\left(\Sigma_{\alpha}^{x^{-1}} \Sigma_{\gamma}^{x}\right)=g_{\alpha \gamma}(x)
$$

Now let us prove ii). We recall how one reconstructs a bundle $F$ starting from the cover $\left\{U_{\alpha}\right\}$ and the coordinate transformations $\left\{g_{\alpha \beta}\right\}$. We have

$$
F=\left(\bigcup_{\alpha}\left(U_{\alpha} \times V\right)\right) / \approx
$$

where for all $(x, v) \in U_{\alpha} \times V,\left(x^{\prime}, v^{\prime}\right) \in U_{\beta} \times V$ we put

$$
(x, v) \approx\left(x^{\prime}, v^{\prime}\right) \quad \text { iff } \quad x=x^{\prime} \quad \text { and } \quad g_{\alpha \beta}(u) v=v^{\prime} .
$$

Define $\widehat{\pi}: F \rightarrow M$ by $\widehat{\pi}[(x, v)]=x$. We denote by $h$ the function defined by

$$
h:\left(\Sigma_{\alpha}^{x}, v\right) \in \Sigma_{m}(M) \times V \mapsto(x, v) \in \bigcup_{\alpha}^{\cdot}\left(U_{\alpha} \times V\right) .
$$


Obviously, $h$ is bijective. Moreover,

$$
\left(\left(\Sigma_{\alpha}^{x}, v\right)\right) \sim\left(\left(\Sigma_{\beta}^{y}, w\right)\right) \Rightarrow x=y, P\left(\Sigma_{\alpha}^{x^{-1}} \Sigma_{\beta}^{x}\right) v=w \Rightarrow x=y, g_{\alpha \beta}(x) v=w \Rightarrow(x, v) \approx(y, w) .
$$

Therefore, we can think of $h$ as defined on the quotient sets

$$
h: \Sigma_{m}(M) \times V / \sim \rightarrow \bigcup_{\alpha}\left(U_{\alpha} \times V\right) / \approx
$$

Since $\widehat{\pi} \circ h=\pi$, we have the required identification. This completes the proof of Lemma 7.1.

Now we show that it is possible to identify the two bundles

$$
F=\Sigma_{m}(M) \times V / \sim \text { and } E:=\tilde{\mathcal{P}}_{m}(M) \times E_{m} / \widetilde{\Omega}_{m}(M) .
$$

Consider the function $l: F \rightarrow E$ given by $l\left\{\left(\Sigma_{\alpha}^{x}, v\right)\right\}:=\left[\left(\Sigma_{\alpha}^{x}, v\right)\right]$. This function is obviously well defined and injective. $l$ is also surjective since for an arbitrary $[(\sigma, v)] \in E$ we may choose an arbitrary $\Sigma_{\alpha}^{x}$ such that $x=f(\sigma)=$ final point of $\sigma$. We have

$$
l\left\{\left(\Sigma_{\alpha}^{x}, P\left(\Sigma_{\alpha}^{x^{-1}} \sigma\right) v\right)\right\}=\left[\left(\Sigma_{\alpha}^{x}, P\left(\Sigma_{\alpha}^{x^{-1}} \sigma\right) v\right)\right]=\left[\left(\left(\Sigma_{\alpha}^{x}\left(\Sigma_{\alpha}^{x^{-1}} \sigma\right), P\left(\Sigma_{\alpha}^{x^{-1}} \sigma\right)^{-1} P\left(\Sigma_{\alpha}^{x^{-1}} \sigma\right) v\right)\right]=[(\sigma, v)] .\right.
$$

So the local trivialization of $F=\Sigma_{m}(M) \times V / \sim$ is indeed a local trivialization of $E:=\widetilde{\mathcal{P}}_{m}(M) \times$ $E_{m} / \widetilde{\Omega}_{m}(M)$.

Step 3: Regularity properties.

In this step we intend to prove that the bundle $E$ is smooth and the same holds for the parallel transport $P: \widetilde{\mathcal{P}}(M) \rightarrow \tau(E)$. Observe that the transition functions of the bundle are defined by

$$
g_{\alpha \beta}(x)=P\left(\Sigma_{\alpha}^{x^{-1}} \Sigma_{\beta}^{x}\right)
$$

and since $P: \widetilde{\Omega}_{m}(M) \rightarrow G$ is smooth by assumption, the differentiability of $g_{\alpha \beta}(x)$ depends on the differentiability of the maps $M \ni x \mapsto \Sigma_{\alpha}^{x^{-1}} \Sigma_{\beta}^{x} \in \Omega_{m}(M)$. It is not difficult to see that any atlas $\left\{U_{\alpha}\right\}_{\alpha \in \mathcal{A}}$ can be equipped with an $m$-contraction $\left\{\Sigma_{\alpha}\right\}_{\alpha \in \mathcal{A}}$ such that the map defined above is smooth. So the $g_{\alpha \beta}(\cdot)$ are smooth. A similar argument holds for parallel transport. This ends the proof of Theorem 7.1.

\section{§8. Some remarks on infinite-dimensional bundle-connection pairs}

The simple algebraic structure of the proof of Theorem 7.1 suggests that a generalization of the theorem could be stated for infinite-dimensional vector bundles. Note that a similar generalization for the AmbroseSinger theorem is still missing (see [35]). The right setting for such a generalization appears to be that of Banach vector bundle over a Hilbert manifold (see [36-40]). Although the notion of a connection has been given also for Frechét vector bundles (see [41]), the correspondence between connections and parallel transports is missing for such bundles because of the "bad" behavior of Frechét spaces with respect to ordinary differential equations ([41-42]). One should also note that in infinite dimension the bijective correspondence between linear connections and covariant derivatives is missing (see [43-39]). In order to add further comments, we give the following definition.

Definition 8.1. A Banach space $F$ is called a Kuiper space if its general linear group $G L(F)$ is contractible (see [44-46]).

\section{Theorem 8.1.}

- Any Hilbert space is a Kuiper space (Kuiper [47]);

- the space $c_{o}$ is a Kuiper space (ArIt [48]);

- the spaces $l_{p}$ are Kuiper spaces (Neubauer [49]). 
Theorem 8.2. $\mathrm{GL}\left(c_{o} \times l_{2}\right)$ is not connected (Douady [50]).

By definition, any vector bundle $E$ with fiber a Kuiper space $F$ and structural group $\mathrm{GL}(F)$ is trivial. Suppose we are given a bundle-connection pair $(E, \nabla)$.

i) The triviality of the bundle $E$ does not imply that the pair $(E, \nabla)$ is trivial: this can happen for geometric reasons, i.e., because of the curvature of the connection $\nabla$, or for topological reasons, i.e., because the base manifold has nontrivial fundamental group.

ii) If the base manifold is infinite-dimensional and the fiber is finite-dimensional, then the theorems of Kuiper, Arlt, Neubauer do not imply the triviality of the bundle.

iii) The result of Douady implies that Banach vector bundles are not globally trivial, in general.

iv) If $F$ is a Banach space, then we denote by $\mathrm{GL}_{c}(F)$ the group of automorphisms of $F$ of the form identity+compact operator. This group is not contractible (see [45]). Hence, a vector bundle with structure group $\mathrm{GL}_{c}(F)$ is not automatically trivial.

It is a pleasure to thank Luigi Accardi, Bruce Driver, and Paolo Roselli for several useful discussions. This research was partially supported by the Post-doctoral fellowship of the University of Pavia.

\section{References}

1. W. Ambrose and I. M. Singer, “A theorem on holonomy," Trans. Amer. Math. Soc., 75, 428-443 (1953).

2. P. W. Michor, Gauge Theory for Fiber Bundles, Bibliopolis, Napoli (1991).

3. C. Ehresmann, "Les connexions infinitésimales dans un espace fibré différentiable," in: Colloque de Topologie, Bruxelles (1950), pp. 29-55.

4. J. Milnor, "On the existence of a connection with curvature zero," Comment. Math. Helv., 32, 215-223 (1958).

5. S. Kobayashi, Differential Geometry of a Complex Vector Bundle, Iwanami Shoten Publishers and Princeton Univ. Press, Princeton (1987).

6. U. G. Lumiste, "Connection theory in bundle space," J. Soviet Math., 1, 363-390 (1973).

7. U. G. Lumiste, "Connection on a fiber bundle," in: Encyclopaedia of Mathematics, Vol. 2, Kluwer Acad. Publ., DordrechtBoston-London (1988), pp. 341-343.

8. S. Kobayashi, "La connexion des variétés fibrées. II," C. $R$. Acad. Sci. Paris, 238, 443-444 (1954).

9. C. Telemann, "Généralisation du groupe fondamental d'un espace topologique," C. R. Acad. Sci. Paris, 248, 2845-2846 (1959).

10. C. Telemann, "Généralisation du groupe fondamental d'une variété différentiable," C. R. Acad. Sci. Paris, 248, 2930-2932 (1959).

11. C. Telemann, "Généralisation du groupe fondamental," Ann. Sci. École Norm. Sup., 77, No. 3, 195-234 (1960).

12. C. Telemann, "Sur la classification des espaces fibrés," C. R. Acad. Sci. Paris, 253, 935-936 (1961).

13. C. Telemann, "Sur les connexions infinitésimales qu'on peut définir dans les structures fibrées différentiables de base donnée," Ann. Mat. Pura Appl., 62, 379-412 (1963).

14. C. Telemann, "Sur une théorie générale des connexions," Bull. Math. Soc. Sci. Math. R. S. Roumanie, 10, No. 1-2, 179-199 (1966).

15. B. K. Driver, "Classification of bundle connection pairs by parallel translation and lassos," J. Funct. Anal., 83, 185-231 (1989).

16. R. Lashof, "Classification of fiber bundles by the loop space of the base," Ann. Math., 64, No. 3, 436-513 (1956).

17. B. Kostant, "Quantization and unitary representations; Prequantization. Part I," in: Lectures in Modern Analysis and Applications III, Vol. 170, Lect. Notes in Math, Springer, Berlin-Heidelberg-New York (1970).

18. R. Giles, "Reconstruction of gauge potentials from Wilson loops," Phys. Rev. D, 24, 2160-2168 (1981).

19. K. G. Wilson, "Confinement of quarks," Phys. Rev. D, 10, 2445-2459 (1974).

20. J. Fröhlich, "Some results and comments on quantized gauge fields," in: Recent Development in Gauge Theories (G. Hooft et al, editors), Plenum, New York (1980).

21. L. Gross, "A Poincaré lemma for connection forms," J. Funct. Anal., 63, 1-46 (1985).

22. B. K. Driver, Loop Space, Curvature and Quasi-Invariance, Preprint No. 123, Centro Vito Volterra, Università di Roma "Tor Vergata" Roma (1992).

23. L. Accardi, P. Gibilisco and I. V. Volovich, "The Lévy Laplacian and the Yang-Mills equations," Rend. Accad. Lincei. Clas. Sci. Fis. Mat. Natur. (9), 4, 201-206 (1993).

24. L. Accardi, P. Gibilisco, and I. V. Volovich, "Yang-Mills gauge fields as harmonic functions for the Lévy Laplacian," Russian J. Math. Phys., 2, 235-250 (1994).

25. F. Speranza, "Sulla nozione di connessione," Boll. Un. Mat. Ital., 20, No. 3, 367-378 (1965).

26. M. V. Mensky, Method of Induced Representations: Space-Time and Concept of Particle [in Russian], Nauka, Moscow (1976).

27. M. V. Mensky, The Group of Paths: Measurements, Fields, Particles [in Russian], Nauka, Moscow (1983).

28. M. V. Mensky, "The path group and the interaction of quantum strings," Soviet Phys. JETP, 63, No. 2 (1986). 
29. L. Accardi and P. Gibilisco, "The Schrödinger representation on Hilbert bundles," in: Probabilistic Methods in Mathematical Physics (F. Guerra, M. I. Loffredo and C. Marchioro, editors), World Scientific, Singapore (1992), pp. 1-15.

30. P. Gibilisco, Representations of Path Groups and Parallel Transports [in Italian], Ph. D. Thesis, Università degli studi di Roma "Tor Vergata" Roma (1992).

31. P. Gibilisco, "The imprimitivity theorem for a class of non-locally compact groups," in: General Relativity and Gravitational Physics (M. Cerdonio, R. D'Auria, M. Francaviglia, and G. Magnano, editors), World Scientific, Singapore (1994), pp. $491-496$.

32. P. Gibilisko, "Induced representations of non-locally compact groups," Mat. Zametki [Math. Notes], 57, No. 3, 350-358 (1995).

33. N. Steenrod, The Topology of Fiber Bundles, Princeton Univ. Press, Princeton, N.J. (1951).

34. S. Kobayashi and K. Nomizu, Foundations of Differential Geometry. Vol. 1, Interscience, New York (1963).

35. D. S. Freed, "The geometry of loop groups," J. Diff. Geom., 28, 223-276 (1988).

36. S. Lang, Differential Manifolds, Springer, Berlin-Heidelberg-New York (1985).

37. H. I. Eliasson, "Geometry of manifolds of maps," J. Diff. Geom., 1, 169-194 (1967).

38. J. Vilms, "Connections on tangent bundles," J. Diff. Geom., 1, 235-243 (1967).

39. W. Klingenberg, Lecture on Closed Geodesic, Springer, Berlin-Heidelberg-New York (1978).

40. W. Klingenberg, Riemannian Geometry, Walter de Gruyter, Berlin-New York (1982).

41. R. Hamilton, "The inverse function theorem of Nash and Moser," Bull. Amer. Math. Soc., 7, 65-222 (1982).

42. J. Milnor, "Remarks on infinite-dimensional Lie groups," in: Relativity, Groups and Topology. II (B. S. De Witt and R. Stora, editors), Les Houches 1983, North-Holland, Amsterdam (1984), pp. 1007-1058.

43. P. Flaschel and W. Klingeberg, Riemannsche Hilbertmannigfaltigkeiten periodische geödetische, Lect. Notes in Math, Vol. 282, Springer, Berlin (1972).

44. Global Analysis (S. S. Chern and S. Smale, editors), Proc. Sympos. Pure Math., Vol. 15, Amer. Math. Soc., Providence, R.I. (1970).

45. U. Koschorke, "Infinite-dimensional K-theory and characteristic classes of Fredholm bundle maps," in: Global Analysis (S. S. Chern and S. Smale, editors), Proc. Sympos. Pure Math., Vol. 15, Amer. Math. Soc., Providence, R.I. (1970), pp. 95-134.

46. K. D. Elworthy and A. J. Tromba, "Differential structures and Fredholm maps on Banach manifolds," in: Global Analysis (S. S. Chern and S. Smale, editors), Proc. Sympos. Pure Math., Vol. 15, Amer. Math. Soc., Providence, R.I. (1970), pp. 45-94.

47. N. H. Kuiper, "The homotopy type of the unitary group of Hilbert spaces," Topology, 3, 19-30 (1965).

48. D. Arlt, "Zusammenziehbarkeit der allgemeinen linearen Gruppe des Raumes co der Nullfolgen," Invent. Math., 1, 36-44 (1966).

49. G. Neubauer, On a class of sequences spaces with contractible linear group, Notes, Univ. of California, Berkeley, California (1967).

50. A. Douady, "Un espace de Banach dont le groupe linéaire n'est pas connexe," Indag. Math., 68, 787-789 (1965).

Dipartamento di Matematica Politecnico di Torino and

Centro "Vito Volterra" Università di Roma "Tor Vergata" 